\title{
Dual Phase-space Cascades in 3D Hybrid-Vlasov-Maxwell Turbulence
}

\author{
S. S. Cerri ${ }^{1}\left(\mathbb{D}\right.$, M. W. Kunz ${ }^{1,2}$ (i) , and F. Califano ${ }^{3}$ (1) \\ ${ }^{1}$ Department of Astrophysical Sciences, Princeton University, Princeton, NJ 08544, USA; scerri@ astro.princeton.edu \\ ${ }^{2}$ Princeton Plasma Physics Laboratory, P.O. Box 451, Princeton, NJ 08543, USA \\ ${ }^{3}$ Dipartimento di Fisica "E. Fermi", Università di Pisa, I-56127 Pisa, Italy \\ Received 2018 February 16; revised 2018 March 6; accepted 2018 March 8; published 2018 March 23
}

\begin{abstract}
To explain energy dissipation via turbulence in collisionless, magnetized plasmas, the existence of a dual real- and velocity-space cascade of ion-entropy fluctuations below the ion gyroradius has been proposed. Such a dual cascade, predicted by the gyrokinetic theory, has previously been observed in gyrokinetic simulations of twodimensional, electrostatic turbulence. For the first time, we show evidence for a dual phase-space cascade of ionentropy fluctuations in a three-dimensional simulation of hybrid-kinetic, electromagnetic turbulence. Some of the scalings observed in the energy spectra are consistent with a generalized theory for the cascade that accounts for the spectral anisotropy of critically balanced, intermittent, sub-ion-Larmor-scale fluctuations. The observed velocity-space cascade is also anisotropic with respect to the magnetic-field direction, with linear phase mixing along magnetic-field lines proceeding mainly at spatial scales above the ion gyroradius and nonlinear phase mixing across magnetic-field lines proceeding at perpendicular scales below the ion gyroradius. Such phase-space anisotropy could be sought in heliospheric and magnetospheric data of solar-wind turbulence and has far-reaching implications for the dissipation of turbulence in weakly collisional astrophysical plasmas.
\end{abstract}

Key words: methods: numerical - plasmas - solar wind - turbulence

\section{Introduction}

Turbulence is one means by which a fluid or plasma nonlinearly transforms kinetic and/or electromagnetic energy into thermodynamic heat. In a fluid or collisional plasma, this conversion is mediated by molecular viscosity and/or resistivity, which dissipatively remove power from small spatial scales. However, many astrophysical and space plasmas are so hot and diffuse that their collisional mean free paths are as large as, if not bigger than, the macroscopic scales of interest. Examples include the solar wind, the intracluster medium, and low-luminosity accretion flows. In this situation, the production of small-scale ("kinetic") structure in velocity space can compensate for the small collisionality and eventually lead to collisional relaxation and irreversible heating. In other words, turbulent collisionless plasmas find a nonlinear route to dissipation through phase space (see Schekochihin et al. 2008).

One way to generate this velocity-space structure is via linear phase mixing, caused by the ballistic response of the particle distribution function and associated with Landau (1946) damping. In a magnetized plasma, this process occurs primarily along magnetic-field lines, being stifled across them by the smallness of particles' gyroradii. Another route to generating small-scale structure in velocity space is nonlinear phase mixing, by which the particles' distribution function at a given position is nonlinearly mixed by decorrelated ring-averaged fluctuations. This mechanism, predicted by the gyrokinetic theory (Dorland \& Hammett 1993; Schekochihin et al. 2008, 2009; Plunk et al. 2010), causes a dual cascade in both the real and velocity spaces perpendicular to the magnetic field of fluctuations in the ion distribution function-the so-called entropy cascade.

Classical turbulence theory is formulated in Fourier space, where nonlinear interactions between fluctuations produce a flux of energy to larger wavenumbers with varying degrees of wavevector anisotropy with respect to the magnetic-field direction (e.g., Iroshnikov 1963; Kraichnan 1965; Goldreich \& Sridhar 1995; Boldyrev 2006). An analogous approach can be used to study velocity-space cascades by instead employing Hermite polynomials or Hankel functions as the basis (e.g., Plunk et al. 2010; Adkins \& Schekochihin 2018, and references therein).

Unfortunately, velocity-space cascades have traditionally been difficult to diagnose, both in satellite data and in numerical simulations. Although several three-dimensional (3D) simulations of kinetic/gyrokinetic turbulence exist (e.g., Howes et al. 2008, 2011; Servidio et al. 2015; Told et al. 2015; Wan et al. 2016; Cerri et al. 2017; Grošelj et al. 2018; L. Arzamasskiy et al. 2018, in preparation; Franci et al. 2018), thus far the velocity-space cascade produced by nonlinear phase mixing has been directly diagnosed only in twodimensional (2D) simulations of gyrokinetic electrostatic turbulence (Tatsuno et al. 2009, 2012), with little other indirect evidence (Bañón Navarro et al. 2011; Cerri et al. 2014). Numerical evidence of linear phase mixing and its associated velocity-space cascade exists, but still within reduced settings (e.g., Watanabe \& Sugama 2004; Hatch et al. 2014; Parker et al. 2016; Grošelj et al. 2017). Observationally, only recent advances in spacecraft instrumentation have provided the first evidence of a velocity-space cascade (in the electron distribution function) occurring in the solar wind filling the Earth's magnetosheath (Servidio et al. 2017).

In this Letter, we report on the occurrence of a phase-space cascade in a high-resolution 3D-3V simulation of hybrid-VlasovMaxwell (HVM) turbulence in a collisionless plasma with finite electron inertia. We also derive scaling laws for the ion-entropy cascade, generalized to account for different spectral anisotropies and a possible reconnection-mediated energy transfer. Some of these scalings are in agreement with our simulation results.

\section{Sub-ion-Larmor Turbulence}

\subsection{Kinetic-Alfvén-wave (KAW) and Ion-entropy Cascades}

As turbulent energy arrives from the "MHD" inertial range at $k_{\perp} \rho_{\mathrm{i}} \sim 1$ ( $k_{\perp}$ is the wavenumber perpendicular to the magnetic 
field, $\rho_{\mathrm{i}}$ is the ion Larmor radius), it can be resonantly absorbed and/or redistributed into various phase-space cascades. Two examples of the latter are those of KAWs and of ion-entropy fluctuations (Schekochihin et al. 2008, 2009; Kunz et al. 2017). By way of reviewing their predicted turbulence scaling laws, we generalize them to account for different wavevector anisotropies.

We measure ion-entropy fluctuations via

$$
\delta \mathcal{E} \equiv-\int d^{3} \boldsymbol{v}\left(f \ln f-F_{\mathrm{M}} \ln F_{\mathrm{M}}\right),
$$

where $F_{\mathrm{M}}=F_{\mathrm{M}}(t, \boldsymbol{r}, \boldsymbol{v})$ is an isotropic Maxwellian whose number density $n$, mean flow $\boldsymbol{u}$, and temperature $T$ matches, respectively, the zeroth, first, and second moments of the ion distribution function $f=f(t, \boldsymbol{r}, \boldsymbol{v})$. Often, a linearized version of Equation (1) is defined, $\delta \mathcal{E} \approx \int d^{3} \boldsymbol{v}\left(T \delta f^{2} / 2 F_{\mathrm{M}}\right)$ with $\delta f \equiv$ $f-F_{\mathrm{M}}$, which features in a collisionless invariant called the gyrokinetic free energy (Schekochihin et al. 2009). For low-frequency, sub-ion-Larmor-scale KAW turbulence, this $\delta f$ is asymptotically equal to the non-adiabatic "gyrokinetic" response $h$, i.e., $\delta f \approx h$ and $\delta \mathcal{E} \approx \int d^{3} \boldsymbol{v}\left(T h^{2} / 2 F_{\mathrm{M}}\right)$ for $k_{\perp} \rho_{\mathrm{i}} \gg 1$. In the gyrokinetic theory, the KAW and ion-entropy cascades are energetically decoupled, with $h$ being a passive tracer of the ring-averaged KAW turbulence in phase space.

As the fluctuations cascade via nonlinear interactions to $k_{\perp} \rho_{\mathrm{i}} \gg 1$, particles with different gyroradii but similar guidingcenter positions-i.e., with different velocities perpendicular to the magnetic field-experience different fluctuations. Plasma particles belonging to different portions of $f$ then undergo different turbulent evolution, leading to phase mixing in the velocity space perpendicular to the magnetic field $\left(v_{\perp}\right)$ with correlation scale

$$
\frac{\delta v_{\perp}}{v_{\text {thi }}} \sim \frac{1}{\rho_{\mathrm{i}}}\left|\frac{v_{\perp}}{\Omega_{\mathrm{i}}}-\frac{v_{\perp}^{\prime}}{\Omega_{\mathrm{i}}}\right| \sim \frac{1}{k_{\perp} \rho_{\mathrm{i}}} \ll 1,
$$

where $v_{\text {thi }} \equiv \sqrt{2 T / m_{\mathrm{i}}}$ is the ion thermal speed, $m_{\mathrm{i}}$ is the ion mass, and $\Omega_{\mathrm{i}}$ is the ion Larmor frequency (see Schekochihin et al. 2009, Section 7.9.1). In the Hermite representation of velocity space, $\delta v_{\perp} / v_{\text {thi }} \sim m_{\perp}^{-1 / 2}$, where $m_{\perp}$ is the order of the Hermite basis function, and so Equation (2) implies $m_{\perp} \propto k_{\perp}^{2}$. The real- and velocity-space cascades of ion-entropy fluctuations are thus tightly entwined and occur simultaneously: ion-entropy fluctuations are mixed in velocity space by decorrelated fluctuations in real space, and the resulting decorrelated velocity-space structure impacts the ring-averaging of those fluctuations. Scaling laws for the entropy cascade should reflect this mixing.

In Section 2.2, we derive such scaling laws using arguments borrowed from gyrokinetic theory. In doing so, we are neither promoting the gyrokinetic theory as a generic description of sub-ion-Larmor-scale turbulence, nor are we claiming that our simulation parameters (see Section 3) are best suited to test such a theory, which is based on a low-frequency, smallamplitude, spatially anisotropic asymptotic ordering. Rather, the agreement or lack thereof between the consequent scaling theory and our simulation results highlights the degree to which certain aspects of the gyrokinetic theory manifest in a more general (Vlasov) setting. It is important to note, however, that the concept of correlated real- and velocity-space fluctuations in the sub-ion-Larmor range (as in Equation (2)) does not require especially low frequencies; it is a generic consequence of particles with similar guiding centers but gyroradii differing by an amount $\sim k_{\perp}^{-1}$ experiencing decorrelated electromagnetic fluctuations.

\subsection{Spectral Anisotropy and Turbulence Scaling Laws}

We denote the scales perpendicular and parallel to the magnetic-field direction by $\lambda$ and $\ell_{\|}$, respectively. The relation between these scales is parameterized as follows:

$$
\ell_{\|, \lambda} \propto \lambda^{\alpha / 3},
$$

where $\alpha$ describes the spectral anisotropy of the fluctuations in the sub-ion-Larmor range of interest. $\alpha=1$ returns the "standard" scalings for KAW turbulence (e.g., Schekochihin et al. 2009; Boldyrev et al. 2013); $\alpha=2$ corresponds to the intermittency-corrected scenario proposed by Boldyrev \& Perez (2012); and $\alpha=3$ describes an "isotropic" cascade, which has been measured in some hybrid-PIC simulations (L. Arzamasskiy et al. 2018, in preparation; Franci et al. 2018).

Using the characteristic timescale of linear KAWs $\left(\tau_{\mathrm{KAW}, \lambda} \propto \lambda \ell_{\|, \lambda}\right)$ and assuming a critically balanced cascade in which the scale-dependent linear and nonlinear timescales are comparable, the nonlinear timescale $\tau_{\mathrm{nl}, \lambda}^{(\alpha)}$ at perpendicular scale $\lambda$ satisfies

$$
\tau_{\mathrm{nl}, \lambda} \sim \tau_{\mathrm{KAW}, \lambda}^{(\alpha)} \propto \lambda^{1+\alpha / 3} .
$$

Following the arguments in (Schekochihin et al. 2009, Section 7.9.2), the nonlinear timescale in the entropy cascade is obtained by weighting $\tau_{\mathrm{KAW}, \lambda}^{(\alpha)}$ by the factor $\left(\rho_{\mathrm{i}} / \lambda\right)^{1 / 2}$ due to the ring-averaging in the gyrokinetic nonlinearity:

$$
\widetilde{\tau}_{h, \lambda} \sim\left(\frac{\rho_{\mathrm{i}}}{\lambda}\right)^{1 / 2} \tau_{\mathrm{KAW}, \lambda}^{(\alpha)}
$$

With $\lambda \ll \rho_{\mathrm{i}}$, during each KAW correlation time the nonlinearity changes the scale-dependent gyrokinetic response $h_{\lambda}$ only by a small factor, $\Delta h_{\lambda} / h_{\lambda} \sim \tau_{\mathrm{KAW}, \lambda}^{(\alpha)} / \widetilde{\tau}_{h, \lambda} \ll 1$. These changes accumulate as a random walk, i.e., as $\left(t / \tau_{\mathrm{KAW}, \lambda}^{(\alpha)}\right)^{1 / 2} \Delta h_{\lambda}$. The entropy cascade time $\tau_{h, \lambda}^{(\alpha)}$ is the time needed to produce an order-unity change in $h_{\lambda}$, i.e., $\left(\tau_{h, \lambda}^{(\alpha)} / \tau_{\mathrm{KAW}, \lambda}^{(\alpha)}\right)^{1 / 2} \Delta h_{\lambda} \sim h_{\lambda}$. Thus,

$$
\tau_{h, \lambda}^{(\alpha)} \sim\left(\frac{\rho_{\mathrm{i}}}{\lambda}\right) \tau_{\mathrm{KAW}, \lambda}^{(\alpha)} \sim \lambda^{\alpha / 3} .
$$

Assuming a constant entropy flux through scales, $h_{\lambda}^{2} / \tau_{h, \lambda} \sim$ $\varepsilon_{h}=$ const, we obtain

$$
h_{\lambda}^{(\alpha)} \propto \lambda^{\alpha / 6},
$$

which corresponds to the following spectra of $h$ and $\delta \mathcal{E}$ :

$$
\begin{aligned}
E_{h}\left(k_{\perp}\right) & \propto k_{\perp}^{-(3+\alpha) / 3}, \\
E_{\delta \mathcal{E}}\left(k_{\perp}\right) & \propto k_{\perp}^{-(3+2 \alpha) / 3} .
\end{aligned}
$$

For standard KAW-turbulence anisotropy $k_{\|} \propto k_{\perp}^{1 / 3}(\alpha=1)$, the predicted $h$ spectrum $\propto k_{\perp}^{-4 / 3}$ is recovered (Schekochihin et al. 2009), and $E_{\delta \mathcal{E}} \propto k_{\perp}^{-5 / 3}$. In the intermittency-corrected case $(\alpha=2), k_{\|} \propto k_{\perp}^{2 / 3}$ and so $E_{h} \propto k_{\perp}^{-5 / 3}$ and $E_{\delta \mathcal{E}} \propto k_{\perp}^{-7 / 3}$. For isotropic sub-ion-scale turbulence ( $\alpha=3), k_{\|} \propto k_{\perp}$ and so $E_{h} \propto k_{\perp}^{-2}$ and $E_{\delta \mathcal{E}} \propto k_{\perp}^{-3}$. In all of these cases, the 


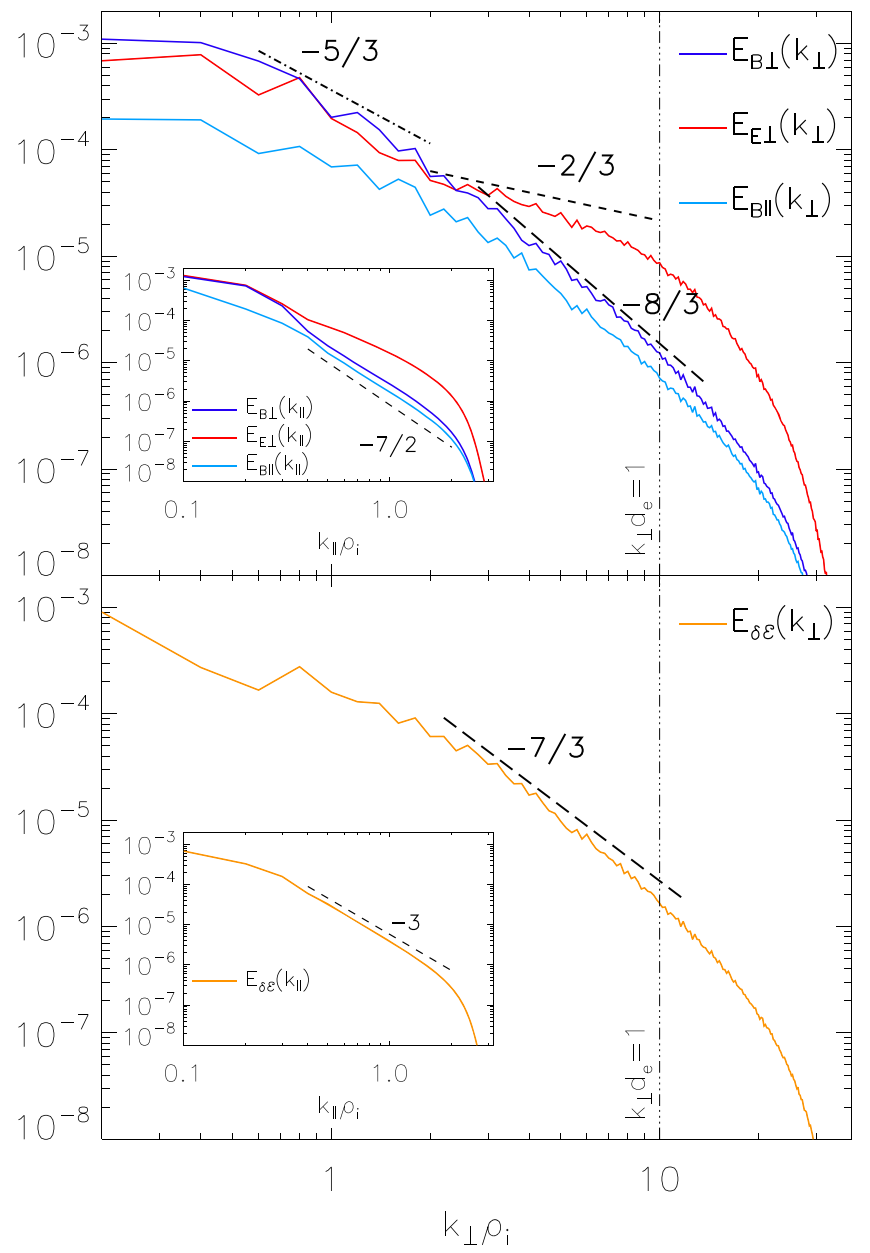

Figure 1. Energy spectra of the perpendicular electric field $E_{E \perp}$ (red solid line), the perpendicular magnetic field $E_{B \perp}$ (blue solid line), the parallel magneticfield fluctuations $E_{B \|}$ (light-blue solid line), and the non-thermal entropy fluctuations $E_{\delta \mathcal{E}}$ (orange solid line), all vs. $k_{\perp}$. Insets report the same spectra vs. $k_{\|}$. Labeled dashed and dotted-dashed lines provide reference slopes.

corresponding spectra in parallel wavenumber are independent of $\alpha$ :

$$
E_{h}\left(k_{\|}\right) \propto k_{\|}^{-2} \quad \text { and } \quad E_{\delta \mathcal{E}}\left(k_{\|}\right) \propto k_{\|}^{-3}
$$

Accompanying these real-space spectra are velocity-space spectra described by the perpendicular Hermite number $m_{\perp}$. Using $m_{\perp} \propto k_{\perp}^{2}$ (see Equation (2)) in Equation (8) implies $E_{h}\left(m_{\perp}\right) \propto m_{\perp}^{-(\overline{6}+\alpha) / 6}$. Note that $E_{h}\left(m_{\perp}\right) \propto m_{\perp}^{-7 / 6}$ for $\alpha=1$, which is equivalent to the $E_{h}(p) \propto p^{-4 / 3}$ Hankel spectrum predicted by Plunk et al. (2010) for nonlinear phase mixing in 2D electrostatic gyrokinetic turbulence (note that $p \sim \sqrt{m_{\perp}}$ ). In the intermittency-corrected case, $E_{h}\left(m_{\perp}\right) \propto m_{\perp}^{-4 / 3}$.

We caution that some of these scalings may be degenerate with a turbulent cascade whose energy transfer is mediated by magnetic reconnection (e.g., Cerri \& Califano 2017; Franci et al. 2017). As the cascade proceeds toward smaller spatial scales and the wavevector anisotropy increases, the reconnection timescale of the turbulent fluctuations may eventually become comparable to the nonlinear cascade time (Loureiro \& Boldyrev 2017; Mallet et al. 2017). As discussed in Mallet et al. (2017), this process will naturally "reset" the wavevector anisotropy and can thus provide an alternative explanation for spectral anisotropies with $\alpha>1$. To explore this possibility, we adopt the scale-dependent reconnection timescale as the nonlinear cascade time for the turbulent cascade upon which the ion-entropy cascade develops; that is, we substitute $\tau_{\text {rec, } \lambda}$ for $\tau_{\mathrm{KAW}, \lambda}$ in the arguments leading to Equations (8) and (9). Using the rates given in Loureiro \& Boldyrev (2017) for $\beta \sim 1$, viz. $\tau_{\text {rec, } \lambda}^{(n)} \propto \lambda^{(4 n+2) /(3 n)}$ (the index $1<n \leqslant 2$ parameterizes cases where the tearing-mode parameter $\Delta^{\prime} \sim \lambda^{-1}(k \lambda)^{-n}$, with $\lambda$ being a proxy for the current-sheet thickness), it is easy to show that $h_{\lambda}^{(n)} \propto \lambda^{(n+2) /(6 n)}$. For the limiting cases $n=1$ and $n=2$, we recover the spectra in Equations (8) and (9) for $\alpha=3$ and $\alpha=2$, respectively. For $n=1$, the reconnection rate, $\propto \lambda^{-2}$, at kinetic scales becomes large enough to efficiently disrupt the turbulent "eddies," producing an effectively isotropic cascade ( $\alpha=3$ ). For $n=2$, such disruption events may occur, but perhaps not so efficiently as to completely isotropize the cascade; sheet-like structures may then persist long enough to produce the intermittency-corrected spectral anisotropy, $\alpha=2$.

\section{Ion-entropy Cascade in HVM Turbulence}

The possibility of a dual phase-space cascade at sub-ionLarmor scales is investigated using a nonlinear simulation of decaying turbulence with the Eulerian (i.e., grid-based both in real and velocity space) HVM code (Valentini et al. 2007). The model equations governing the ion distribution function $f(t, \boldsymbol{r}, \boldsymbol{v})$ and the electromagnetic fields $\boldsymbol{E}(t, \boldsymbol{r})$ and $\boldsymbol{B}(t, \boldsymbol{r})$ are the Vlasov equation, Faraday's law of induction, and a generalized Ohm's law that assumes quasi-neutrality and includes the inductive and Hall electric fields, a thermo-electric field driven by pressure gradients in the (isothermal) electron fluid, and the leading-order electron inertia term (Cerri et al. 2017):

$$
\begin{gathered}
\frac{\partial f}{\partial t}+\boldsymbol{v} \cdot \nabla f+(\boldsymbol{E}+\boldsymbol{v} \times \boldsymbol{B}) \cdot \frac{\partial f}{\partial \boldsymbol{v}}=0 \\
\left(1-d_{\mathrm{e}}^{2} \nabla_{\perp}^{2}\right) \boldsymbol{E}=-\boldsymbol{u} \times \boldsymbol{B}+\frac{(\boldsymbol{\nabla} \times \boldsymbol{B}) \times \boldsymbol{B}}{n}-\frac{T_{\mathrm{e}} \boldsymbol{\nabla} n}{n}, \\
\frac{\partial \boldsymbol{B}}{\partial t}=-\boldsymbol{\nabla} \times \boldsymbol{E}
\end{gathered}
$$

where all quantities are normalized using $m_{\mathrm{i}}, \Omega_{\mathrm{i}}$, the Alfvén speed $v_{\mathrm{A}} \equiv B / \sqrt{4 \pi m_{\mathrm{i}} n}$, and the ion inertial length $d_{\mathrm{i}} \equiv v_{\mathrm{A}} / \Omega_{\mathrm{i}}$. The electron inertia term in Equation (12) involving the electron inertial length $d_{\mathrm{e}}$ is the only term able to physically break flux freezing and allow magnetic reconnection to occur. To correctly capture such physics, a reduced mass ratio $m_{\mathrm{i}} / m_{\mathrm{e}}=100$ has been adopted so that $d_{\mathrm{e}}=0.1 d_{\mathrm{i}}$ is spatially resolved.

The simulation (published in Cerri et al. 2017) was initialized with a stationary, spatially homogeneous, Maxwellian, ionelectron plasma, threaded by a uniform magnetic field $\boldsymbol{B}_{0}=B_{0} \hat{\boldsymbol{z}}$ and characterized by a plasma beta parameter $\beta_{\mathrm{i} 0}=\beta_{\mathrm{e} 0} \equiv$ $8 \pi n_{0} T_{0} / B_{0}^{2}=1$ (the subscript " 0 " denotes an initial value). Large-scale, random, nearly isotropic 3D magnetic perturbations $\delta \boldsymbol{B}$ are placed on top of $\boldsymbol{B}_{0}$, with $0.1 \leqslant\left(k \rho_{\mathrm{i}}\right)_{\delta \boldsymbol{B}} \leqslant 0.5$ and $\delta$ $B_{\text {rms }} \simeq 0.23$. The ion phase space is discretized using $\left(N_{x}, N_{y}, N_{z}, N_{v_{x}}, N_{v_{y}}, N_{v_{z}}\right)=(384,384,64,51,51,51)$ uniformly distributed points. Periodic boundary conditions are imposed in real space, with $L_{z} \simeq 62 \rho_{\mathrm{i}}$ and $L_{x}=L_{y} \simeq 31 \rho_{\mathrm{i}}$, corresponding to a spectral domain spanning $0.2 \leqslant k_{x, y} \quad \rho_{\mathrm{i}} \leqslant 38.4$ and 
$0.1 \leqslant k_{z} \rho_{\mathrm{i}} \leqslant 3.2$. Velocity space is limited in each direction by $v_{\max }= \pm 5 \sqrt{T_{0} / m_{\mathrm{i}}}$, beyond which $f=0$.

The initial $\delta \boldsymbol{B}$ freely decays into fully developed turbulence after a few Alfvén-crossing times, when a peak in the rms current density and quasi-stationary energy spectra are obtained. Our analysis is performed during this fully developed turbulent state, which is characterized by intermittent magneticfield fluctuations across and below $\rho_{\mathrm{i}}$ and by a spectral anisotropy that, in the $k_{\perp} \rho_{\mathrm{i}} \gtrsim 1$ kinetic range, is consistent with this intermittency, viz., $k_{\|} \propto k_{\perp}^{2 / 3}$.

\subsection{Real-space Cascades}

The top panel of Figure 1 displays energy spectra of the perpendicular electric field $E_{E \perp}$ (red solid line), the perpendicular magnetic field $E_{B \perp}$ (blue solid line), and the parallel magnetic-field fluctuations $E_{B \|}$ (light-blue solid line) versus $k_{\perp}$. The inset shows the same spectra versus $k_{\|}$. (The parallel electric-field spectrum, not shown, is at least an order of magnitude smaller than any other spectrum.) Here, " $\|$ " and " $\perp$ " are defined with respect to $\boldsymbol{B}_{0}$. Both $E_{B \perp}$ and $E_{B \|}$ exhibit a break near $k_{\perp} \rho_{\mathrm{i}} \approx 2$, with a sub-ionLarmor-scale slope close to $-8 / 3$. The perpendicular electric-field spectrum at these scales is shallower, with slope initially close to $-2 / 3$ but progressively steepened by electron inertial effects. The spectral cutoffs at $k_{\perp} \rho_{\mathrm{i}} \gtrsim 20$ and $k_{\|} \rho_{\mathrm{i}} \gtrsim 2$ are caused by electron inertia and (weak) spectral filters.

The bottom panel of Figure 1 displays $E_{\delta \mathcal{E}}\left(k_{\perp}\right)$; the inset shows $E_{\delta \mathcal{E}}\left(k_{\|}\right)$. In the kinetic range, below the scales at which the magnetic-field spectrum breaks, the $\delta \mathcal{E}$ spectrum shows a spectral slope near $-7 / 3$. The corresponding $k_{\|}$spectrum has a slope very close to -3 . Both are in remarkable agreement with the theory presented in Section 2.2 for $\alpha=2$, derived by treating $\delta \mathcal{E}$ as a passive scalar of intermittent KAW turbulence.

\subsection{Phase-space Representation of the Cascade}

The ion-entropy cascade is predicted to be a dual cascade simultaneously in real and velocity space. To test this idea, we must Fourier-Hermite transform $\delta f$. However, due to computational expense, the full 3D-3V distribution function spanning the entire computational domain cannot be analyzed at once. Instead, we have analyzed two reduced quantities separately: (i) a $v_{z}$-integrated $\delta f$ measured at eight different $z$ locations, $\left\langle\delta f\left(x, y, z=\left\{z_{0}\right\}_{j=1 \ldots 8}, v_{x}, v_{y}\right)\right\rangle_{v_{z}}$; and (ii) a $\left(v_{x}, v_{y}\right)$-integrated $\delta f$ with no spatial information removed, $\left\langle\delta f\left(x, y, z, v_{z}\right)\right\rangle_{v_{x}, v_{y}}$. These quantities are Fourier-Hermite transformed using a maximum Hermite mode number $M=30$ (accounting for our finite velocity-space resolution). The first reduced quantity then gives the spectrum in the $\left(k_{\perp}, m_{\perp}\right)$ plane, while the second is representative of the $\left(k_{\perp}, k_{\|}, m_{\|}\right)$space. Because of the Fourier transform, here " $\|$ " and " $\perp$ " are defined with respect to $\boldsymbol{B}_{0}$, so that $m_{\|}=m_{z}$ and $m_{\perp}=\left(m_{x}^{2}+m_{y}^{2}\right)^{1 / 2}$.

Figure 2 displays contour plots of these reduced phase-space spectra in the $\left(k_{\perp}, k_{\|}\right),\left(k_{\perp}, m_{\perp}\right)$, and $\left(k_{\perp}, m_{\|}\right)$planes. We observe several interesting features. The entropy cascade in real space develops a spectral anisotropy consistent with $k_{\|} \propto k_{\perp}^{2 / 3}$ (panel (a)), the same as occurs in the electromagnetic fields (Cerri et al. 2017). Note that within the hybrid-kinetic model and in the wavenumber range explored by our simulation, effects ordered out of gyrokinetics (e.g., finite Larmor frequency) may be present. Indeed, the white solid lines in Figure 2(a), which trace $\omega_{\mathrm{KAW}} / \Omega_{\mathrm{i}}=0.25,0.5$, and 1 isocontours, indicate that the linear KAW frequency $\omega_{\mathrm{KAW}}$

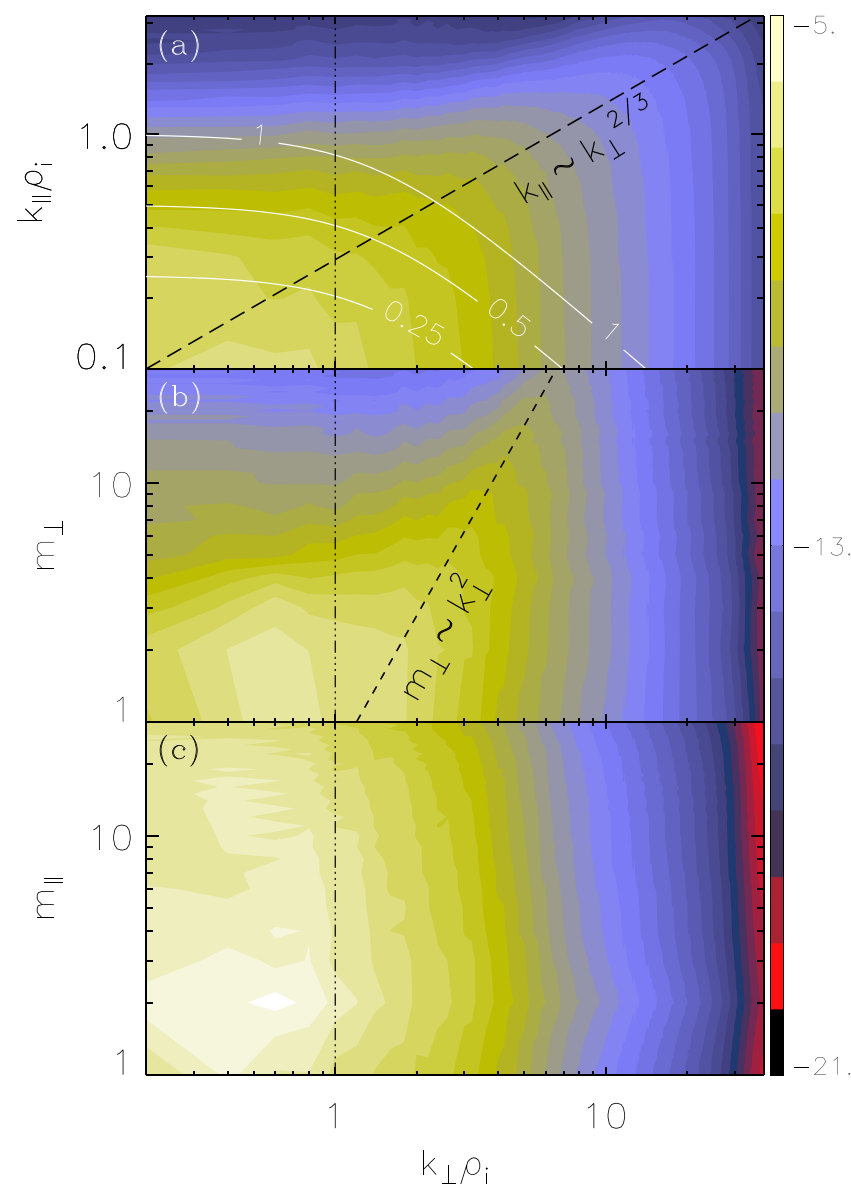

Figure 2. Fourier-Hermite spectrum of the (reduced) non-thermal distribution function, $\log \left|\delta f_{k, m}\right|^{2}$. Top to bottom: $\left(k_{\|}, k_{\perp}\right),\left(m_{\perp}, k_{\perp}\right)$, and $\left(m_{\|}, k_{\perp}\right)$ planes. Labeled dashed lines provide reference slopes. White solid lines in panel (a) trace $\omega_{\mathrm{KAW}} / \Omega_{\mathrm{i}}=0.25,0.5$, and 1 isocontours.

can exceed $\Omega_{\mathrm{i}}$ in our simulation. ${ }^{4}$ Nevertheless, perpendicular phase mixing occurs at $k_{\perp} \rho_{\mathrm{i}} \gtrsim 1$ and consists of a dual phasespace cascade, occurring approximately along $m_{\perp} \propto k_{\perp}^{2}$ (panel (b)). A similar calculation of the Fourier-Hankel spectrum of $\delta f$ (not shown) reveals $p \propto k_{\perp}$, as predicted by Plunk et al. (2010) and consistent with $m_{\perp} \propto k_{\perp}^{2}$. (Using a gyro-averaged $\delta f$ does not qualitatively change these results.) Parallel phase mixing occurs simultaneously, but is confined mainly to $k_{\perp} \rho_{\mathrm{i}} \lesssim 1$ (panel (c)) and $k_{\|} \rho_{\mathrm{i}} \lesssim 0.5$ (not shown), in agreement with the conjecture by (Schekochihin et al. 2009, Section 7.9.4) that nonlinear perpendicular phase mixing is more efficient than linear parallel phase mixing at sub-ion-Larmor scales.

\subsection{Velocity-space Cascades}

We now examine the velocity-space spectrum of $\delta f$. We extracted $\delta f\left(x, y, z, v_{x}, v_{y}, v_{z}\right)$ from eight reduced local subdomains of spatial size $\ell_{x}=\ell_{y} \simeq \rho_{\mathrm{i}}$ and $\ell_{z} \simeq 7 \rho_{\mathrm{i}}$, each consisting of $12 \times 12 \times 8$ grid points, and then performed a 3D Hermite transform at each grid point. Using the local magnetic-field direction $\hat{\boldsymbol{b}} \equiv \boldsymbol{B} / B$, the resulting spectra were transformed

\footnotetext{
4 For simplicity, we have used the approximate expression $\omega_{\mathrm{KAW}}=$ $k_{\|} v_{\mathrm{A}} \sqrt{1+0.5\left(k_{\perp} \rho_{\mathrm{i}}\right)^{2}}$ for $\beta_{\mathrm{i}}=T_{\mathrm{i}} / T_{\mathrm{e}}=1$, which matches the result from the linear gyrokinetic theory to within $\approx 2 \%$ in the wavenumber regime of interest.
} 

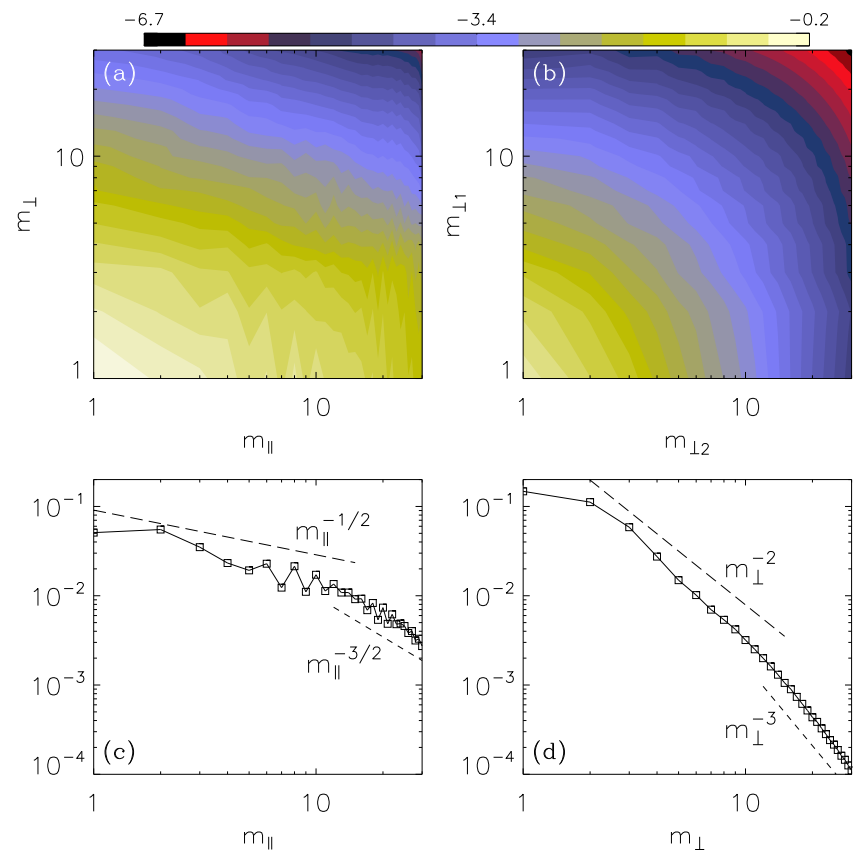

Figure 3. Spatially averaged Hermite spectrum of the non-thermal distribution function, $\log \left|\delta f_{m}\right|^{2}$, in a local magnetic-field-aligned coordinate system $\left(m_{\|}, m_{\perp}\right)$ with $m_{\perp}=\left(m_{\perp 1}^{2}+m_{\perp 2}^{2}\right)^{1 / 2}$. Labeled dashed lines provide reference slopes.

into a field-aligned coordinate system $\left(m_{\perp 1}, m_{\perp 2}, m_{\|}\right)$with $m_{\perp}=$ $\left(m_{\perp 1}^{2}+m_{\perp 2}^{2}\right)^{1 / 2}$.

The resulting (spatially averaged) Hermite spectrum of $\delta f$ is shown in Figure 3..$^{5}$ The spectrum is clearly anisotropic and gyrotropic with respect to $\hat{\boldsymbol{b}}$ (panels (a) and (b)), as expected for magnetized turbulence (e.g., Servidio et al. 2017). The $m_{\|}^{-1 / 2}$ spectrum in Figure 3(c) for $m_{\|} \lesssim 15$ likely reflects linear phase mixing occurring along magnetic-field lines, as predicted by Zocco \& Schekochihin (2011) and Kanekar et al. (2015) (cf. Watanabe \& Sugama 2004). The $m_{\perp}$ spectrum, on the other hand, is noticeably steeper than predicted, being closer to -2 (for $m_{\perp} \lesssim 15$ ). The same $m_{\perp}^{-2}$ spectrum is consistently recovered when integrating the $\delta f$ spectrum in Figure 2(b) over $k_{\perp}$; an analogous procedure applied to the Fourier-Hankel transform of $\delta f$ returns a compatible spectrum close to $p^{-3}$. Likewise, integrating the spectrum in Figure 2(b) over $m_{\perp}$ yields a spectrum close to $k_{\perp}^{-3}$ in the range where the dual cascade is observed, also steeper than our prediction for $E_{h}\left(k_{\perp}\right)$ (see Equation (8)). These results hold true for the gyroaveraged $\delta f$ as well. While we have no explanation for this discrepancy, we do note that Servidio et al. (2017) predict an $m^{-2}$ spectrum and $m$-space anisotropy when magnetic-field fluctuations play a dominant role in the velocity-space cascade (by contrast with the gyrokinetic case, in which the $v_{\perp}$ cascade is driven predominantly by electric-field fluctuations). The difference may be a result of the relatively weak spectral anisotropy in our simulation, which causes the linear KAW frequency to approach $\Omega_{\mathrm{i}}$ somewhat early in the sub-ion-

\footnotetext{
Despite $\delta f$ having no zeroth, first, or second moment, the Hermite spectra exhibit residual $m=1$ and $m=2$ coefficients. There are two causes of this: (i) a finite velocity-space resolution is used when subtracting off the local, shifted Maxwellian $F_{\mathrm{M}}$ from $f$ and then projecting $\delta f$ onto the discrete Hermite basis; and (ii) $F_{\mathrm{M}}$ is taken to be isotropic in velocity space, even though magneticfield-biased temperature anisotropy $T_{\|} \neq T_{\perp} \neq T$ is observed.
}

Larmor range (see Figure 2), violating the gyrokinetic ordering used in Section 2 to predict the $\mathcal{E}_{h}$ spectrum.

Both the parallel and perpendicular Hermite spectra steepen at $m_{\|, \perp} \approx 15$ to approximately $m_{\|}^{-3 / 2}$ and $m_{\perp}^{-3}$. Although a $-3 / 2$ slope is predicted by Adkins \& Schekochihin (2018) and Servidio et al. (2017) for the regime of Vlasov turbulence where advection or electric-field fluctuations dominate, because of the finite velocity-space resolution in our simulation, our measured slopes in this range may not be converged.

\section{Conclusions}

Using a high-resolution HVM simulation, we have shown for the first time that a dual phase-space cascade is occurring in 3D-3V electromagnetic turbulence. Generalized scaling laws that account for different spectral anisotropies with respect to the magnetic-field direction have been derived, and the observed ion-entropy cascade is consistent with some of these new scalings in the intermittency-corrected case, $k_{\|} \propto k_{\perp}^{2 / 3}$ (Boldyrev \& Perez 2012). The non-thermal distribution function $\delta f$ is shown to develop both real- and velocity-space structure that is anisotropic with respect to the magnetic-field direction. Both the real-space cascades of $\delta f$ and the electromagnetic fields exhibit a sub-ion-Larmor-scale spectral anisotropy consistent with $k_{\|} \propto k_{\perp}^{2 / 3}$. At the largest "fluid" scales, $k_{\perp} \rho_{\mathrm{i}} \lesssim 1$, linear phase mixing drives a velocity-space cascade in the Hermite moments along the magnetic-field direction, with a power law close to $m_{\|}^{-1 / 2}$. A dual phase-space cascade in $\left(k_{\perp}, m_{\perp}\right)$ develops at scales below the ion gyroradius, $k_{\perp} \rho_{\mathrm{i}} \gtrsim 1$, with $m_{\perp} \propto k_{\perp}^{2}$ likely due to nonlinear phase mixing (Schekochihin et al. 2008, 2009). The resulting Hermite spectrum is steeper than our predictions based on gyrokinetic theory, but is close to the $m_{\perp}^{-2}$ spectrum predicted for magnetized turbulence in a Vlasov plasma (Servidio et al. 2017). This steepness could be due to effects ordered out of the gyrokinetic theory, such as finite Larmor frequency (see, e.g., Bruno \& Trenchi 2014; Telloni et al. 2015), and/or due to multiscale dissipation or non-local couplings in phase space (e.g., Cerri et al. 2014; Hatch et al. 2014; Passot \& Sulem 2015; Teaca et al. 2017).

These results constitute the first evidence that both linear and nonlinear phase mixing are at play in magneto-kinetic plasma turbulence. Because the resulting spectral scaling laws are largely consistent with theories accounting for turbulent intermittency, we expect the consequent dissipation to be associated with intermittent structures such as current sheets and coherent magnetic structures. This, alongside the fact that reconnection seems to enhance/trigger the energy transfer below ion kinetic scales (Cerri \& Califano 2017; Franci et al. 2017), may explain why most of the kinetic activity, energy conversion, and dissipation in solar-wind turbulence and in kinetic simulations is concentrated within or in the vicinity of ion-scale current sheets (e.g., Osman et al. 2012a, 2012b; Servidio et al. 2012, 2015; Karimabadi et al. 2013; TenBarge \& Howes 2013; Wu et al. 2013; Chasapis et al. 2015; Wan et al. 2015, 2016; Bañón Navarro et al. 2016; Yang et al. 2017). Although the present model neglects possible contributions from electron kinetics at sub-ion-Larmor scales (e.g., Told et al. 2015), we believe these ion-phase-space cascades constitute an important pathway to turbulent dissipation in collisionless plasmas. 
The authors thank Lev Arzamasskiy, Yuri Cavecchi, and especially Alex Schekochihin for valuable conversations, as well as the anonymous referee for a prompt and constructive report. S.S.C. and F.C. acknowledge Carlo Cavazzoni (CINECA, Italy) for essential contributions to the parallelization and optimization of the HVM code. The simulation was performed at CINECA (Italy) under the ISCRA initiative (grant HP10BEANCY). S.S.C. and M.W.K. were supported by the National Aeronautics and Space Administration under grant No. NNX16AK09G issued through the Heliophysics Supporting Research Program.

\section{ORCID iDs}

S. S. Cerri (ib https://orcid.org/0000-0003-0562-6574

M. W. Kunz (iD https://orcid.org/0000-0003-1676-6126

F. Califano (iD https://orcid.org/0000-0002-9626-4371

\section{References}

Adkins, T., \& Schekochihin, A. A. 2018, JPIPh, 84, 905840107

Bañón Navarro, A., Morel, P., Albrecht-Marc, M., et al. 2011, PhRvL, 106, 055001

Bañón Navarro, A., Teaca, B., Told, D., et al. 2016, PhRvL, 117, 245101

Boldyrev, S. 2006, PhRvL, 96, 115002

Boldyrev, S., Horaites, K., Xia, Q., \& Perez, J. C. 2013, ApJ, 777, 41

Boldyrev, S., \& Perez, J. C. 2012, ApJL, 758, L44

Bruno, R., \& Trenchi, L. 2014, ApJL, 787, L24

Cerri, S. S., Bañón Navarro, A., Jenko, F., \& Told, D. 2014, PhPl, 21, 082305

Cerri, S. S., \& Califano, F. 2017, NJPh, 19, 025007

Cerri, S. S., Servidio, S., \& Califano, F. 2017, ApJL, 846, L18

Chasapis, A., Retinò, A., Sahraoui, F., et al. 2015, ApJL, 804, L1

Dorland, W., \& Hammett, G. W. 1993, PhFlB, 5, 812

Franci, L., Cerri, S. S., Califano, F., et al. 2017, ApJL, 850, L16

Franci, L., Landi, S., Verdini, A., Matteini, L., \& Hellinger, P. 2018, ApJ, 853, 26 Goldreich, P., \& Sridhar, S. 1995, ApJ, 438, 763

Grošelj, D., Cerri, S. S., Bañón Navarro, A., et al. 2017, ApJ, 847, 28

Grošelj, D., Mallet, A., Loureiro, N. F., \& Jenko, F. 2018, PhRvL, 120, 105101

Hatch, D. R., Jenko, F., Bratanov, V., \& Bañón Navarro, A. 2014, JPIPh, 80,531

Howes, G. G., Dorland, W., Cowley, S. C., et al. 2008, PhRvL, 100, 065004
Howes, G. G., Tenbarge, J. M., Dorland, W., et al. 2011, PhRvL, 107, 035004 Iroshnikov, R. S. 1963, AZh, 40, 742

Kanekar, A., Schekochihin, A. A., Dorland, W., \& Loureiro, N. F. 2015, JPIPh, 81, 305810104

Karimabadi, H., Roytershteyn, V., Wan, M., et al. 2013, PhPl, 20, 012303

Kraichnan, R. H. 1965, PhFl, 8, 1385

Kunz, M. W., Abel, I. G., Klein, K. G., \& Schekochihin, A. A. 2017, JPIPh, submitted (arXiv:1712.02269)

Landau, L. D. 1946, Zh. Eksp. Teor. Fiz., 16, 574

Loureiro, N. L., \& Boldyrev, S. 2017, ApJ, 850, 182

Mallet, A., Schekochihin, A. A., \& Chandran, B. D. G. 2017, JPIPh, 83, 905830609

Osman, K. T., Matthaeus, W. H., Hnat, B., \& Chapman, S. C. 2012a, PhRvL, 108,261103

Osman, K. T., Matthaeus, W. H., Wan, M., \& Rappazzo, A. F. 2012b, PhRvL, 108,261102

Parker, J. T., Highcock, E. G., Schekochihin, A. A., \& Dellar, P. J. 2016, PhPl, 23, 070703

Passot, T., \& Sulem, P. L. 2015, ApJL, 812, L37

Plunk, G. G., Cowley, S. C., Schekochihin, A. A., \& Tatsuno, T. 2010, JFM, 664,407

Schekochihin, A. A., Cowley, S. C., Dorland, W., et al. 2008, PPCF, 50, 124024

Schekochihin, A. A., Cowley, S. C., Dorland, W., et al. 2009, ApJS, 182 310

Servidio, S., Chasapis, A., Matthaeus, W. H., et al. 2017, PhRvL, 119, 205101 Servidio, S., Valentini, F., Califano, F., \& Veltri, P. 2012, PhRvL, 108, 045001

Servidio, S., Valentini, F., Perrone, D., et al. 2015, JPIPh, 81, 325810107

Tatsuno, T., Dorland, W., Schekochihin, A. A., et al. 2009, PhRvL, 103, 015003

Tatsuno, T., Plunk, G. G., Barnes, M., et al. 2012, PhPl, 19, 122305

Teaca, B., Jenko, F., \& Told, D. 2017, NJPh, 19, 045001

Telloni, D., Bruno, R., \& Trenchi, L. 2015, ApJ, 805, 46

TenBarge, J. M., \& Howes, G. G. 2013, ApJL, 771, L27

Told, D., Jenko, F., TenBarge, J. M., Howes, G. G., \& Hammett, G. W. 2015 PhRvL, 115, 025003

Valentini, F., Trávníček, P., Califano, F., Hellinger, P., \& Mangeney, A. 2007, $\mathrm{JCoPh}, 225,753$

Wan, M., Matthaeus, W. H., Roytershteyn, V., et al. 2015, PhRvL, 114, 175002

Wan, M., Matthaeus, W. H., Roytershteyn, V., et al. 2016, PhPl, 23, 042307

Watanabe, T.-H., \& Sugama, H. 2004, PhPl, 11, 1476

Wu, P., Perri, S., Osman, K., et al. 2013, ApJL, 763, L30

Yang, Y., Matthaeus, W. H., Parashar, T. N., et al. 2017, PhPl, 24, 072306

Zocco, A., \& Schekochihin, A. A. 2011, PhPl, 18, 102309 